\title{
Torn from the headlines: role of public awareness and bench- to- bedside research in prevention and treatment of Acanthamoeba keratitis
}

\author{
Abdul Mannan Baig (1) ${ }^{1}$ \\ Received: 22 October 2018 / Accepted: 14 November 2018 / Published online: 14 December 2018 \\ (c) The Royal College of Ophthalmologists 2018
}

Corneal infection caused by Acanthamoeba spp., with or without the use of contact lenses, is considered to be a sporadic corneal disease. However, once the Acanthamoeba keratitis (AK) sets in, the vision of the patient is threatened and blindness occurs as a consequence in untreated cases. Because of the rare occurrences of AK worldwide, there has been little, if any, interest in the past in raising awareness for prevention of this vision-threatening keratitis in the public in general and contact lens wearers in particular. The latter group of individuals are, in particular, more likely to be victims of AK. Recently, the details of $\mathrm{AK}$ and its potential to cause blindness have captured the attention of international media and news outlets because of a sudden surge in the incidence of AK in the United Kingdom [1] (Fig. 1a).

The precise reason why AK has almost tripled since the year 2011 in the southeast of England remains unknown. This intensifying trend of AK is alarming and disturbing to ophthalmologists, as there are only few biocides and drugs available to treat AK. The non-contact lens-related keratitis epidemic remains a real possibility, as reflected by reports from India where in between 1988 and 2009, only three cases $(0.9 \%)$ of AK occurred among contact lens wearers, whereas 38,529 atypical cases of infectious keratitis were studied with 372 being positive on culture for Acanthamoeba [2]. As related to the successful treatment of AK, it has been observed that advanced $\mathrm{AK}$ patients require treatment for prolonged durations that usually extends to a period of a year in around $70 \%$ of the cases, while in the remaining cases, it extends to a period of more than a year [1]. The recent rise in AK in the United Kingdom in general and southeast of England in particular is an example when a

Abdul Mannan Baig

abdul.mannan@aku.edu

1 The Aga Khan University, Karachi, Pakistan disease that is thought to be infrequent shows its ominous impact on human health by causing disability. The aforementioned regional epidemic in the United Kingdom and similar occurrences in keratitis in the past in Asian countries $[1,2]$, implore the need of extensive research into exploration of risk factors in $\mathrm{AK}$.

With this recent upsurge and similar incidences in the United States, Acanthamoeba spp. has exhibited the potential to induce $\mathrm{AK}$ of endemic proportion that causes blindness if not treated early in the course of the disease. Although it is encouraging to see that the leading electronic media and medical news website resources have turned to highlight this rare type of ocular disease (Fig. 1a), raising public awareness and regularly updating the healthcare professionals on the latest research in $\mathrm{AK}$ is necessary to prevent the occurrence of a large-scale epidemic in the future. The fact that very few people who wear contact lenses know that showering, facewashing, and swimming in warm waters while wearing contact lenses are also likely to be risk factors [1], there is a chance that cases of $\mathrm{AK}$ will continue to rise in the near future. Acanthamoeba spp. is known to affect immunocompromised patients and patients with increased susceptibility to infections. Patients with systemic illness, diabetes mellitus, and recipients of corticosteroids are needed to be studied indepth to identify yet unknown risk factors for AK. There is a need to encourage contact lens users to prefer either daily disposable contact lenses or to develop contact lens cleaning liquids admixed with an eye-friendly amoebicidal and cysticidal drug, to prevent AK. Though more disabling than lethal, a successful treatment of AK has remained a challenge for ophthalmologists worldwide.

\section{Strengths of benchside research in AK}

Basic science research into the biology and pathogenesis of this protist pathogen coupled with research on in vitro effects 


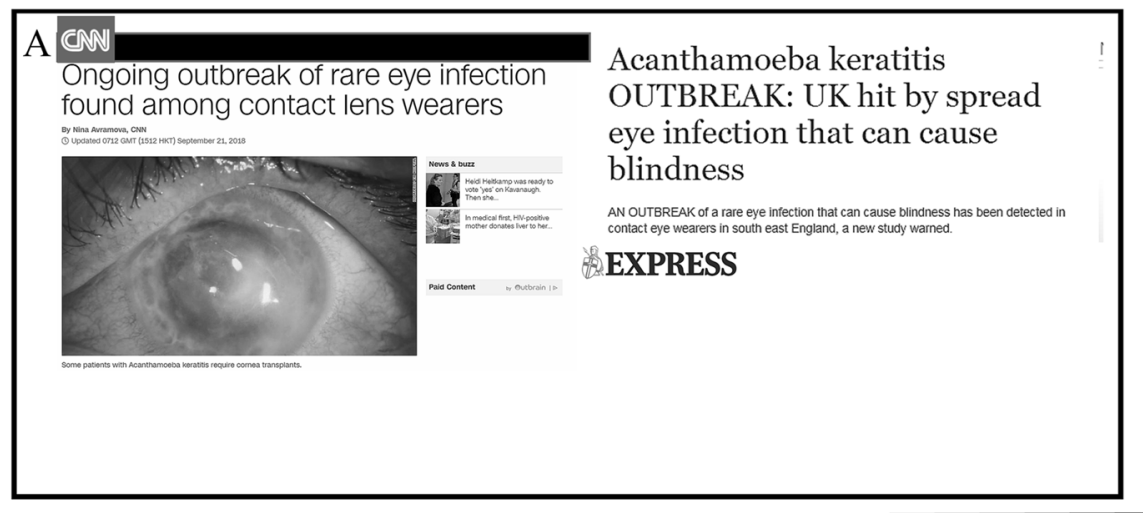

B
Novel amoebicidal chemical agents and

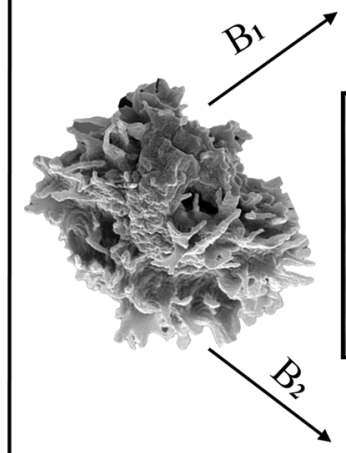
biocides

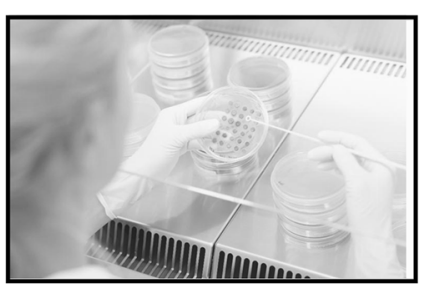

Benchside Amoebicidal drugs already in clinical use

Animal model testing if
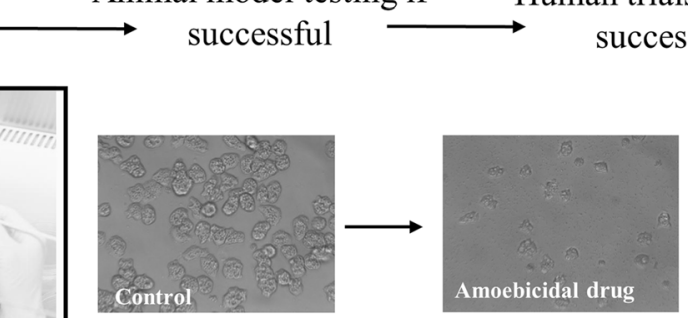

Drug clears efficacy and safety!

Approval for use in $\mathrm{AK}$ Human trials in AK if successful

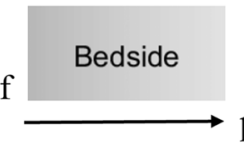

They skip drug efficacy and human safety studies
Fig. 1 a Shows recent mention of Acanthamoeba keratitis (AK) in leading news media and news outlets after an outbreak of AK in the United Kingdom. A similar epidemic of AK has occurred in the United States in the past. b Shows the superiority of FDA-approved drugs that have proven to kill amoebal trophozoites and cysts over novel

of drugs in Acanthamoeba spp., can contribute towards our fight against AK. Subsequently, the drugs that are seen to produce significant amoebicidal and/or cysticidal effects [3] consistently could be taken to the next level of animal testing. Studies on the animal model of AK that uses mammalian species, would be of a more translational value, as they could show the effects of the in vitro-tested drugs on not only Acanthamoeba spp., but also their effects on cornea and the adjacent ocular tissue. The discovery of novel chemicals and biocides for AK, although exciting, faces the challenge of not only to pass the above two phases of testing, but also to clear its safety and efficacy tests in human trials [1]. As the human trails and data gathering is time consuming, it appears that a novel drug and newer biocides with significant amoebicidal and cysticidal effects would not be able to make it to the bedside of patients with AK speedily (Fig. 1-B1). Recently, some drugs that bind and inactivate vital cellular proteins like calmodulin and evolutionary conserved ligands and GPCRs [4] have shown in vitro cysticidal and amoebicidal effects in Acanthamoeba T4 genotype known to cause AK [3]. With the chemicals and biocides (B1). The FDA-approved drugs (see text) would need to be tested only in human eye, as their safety studies have been completed in the past (B2). Note the effects of one of the FDAapproved drugs on Acanthamoeba trophozoites as compared to control

reports of the evidence of the molecular targets of the reported drugs like procyclidine, loperamide, amlodipine and prochlorperazine [3] in Acanthamoeba spp., only testing in the human eye with AK for safety can bring some of them to the bedside (Fig. 1-B2) in a shorter period of time. If a drug that is already approved by the FDA exhibits acanthamoebicidal and cysticidal attributes that turn out to be safe in the human eye, it would not need to pass the drug safety and data-gathering period (Fig. 1-B2), for which a novel drug takes an average of 7-10 years (Fig. 1-B1) before it gets a formal approval for its use in humans.

\section{Strengths of bedside research and trials: role of the ophthalmologists}

For a drug to be significantly successful in AK, it has to establish its clinical efficacy over the current drug regimen in clinical trials and at the same time should ideally be devoid of adverse effects on ocular tissue. The role the 


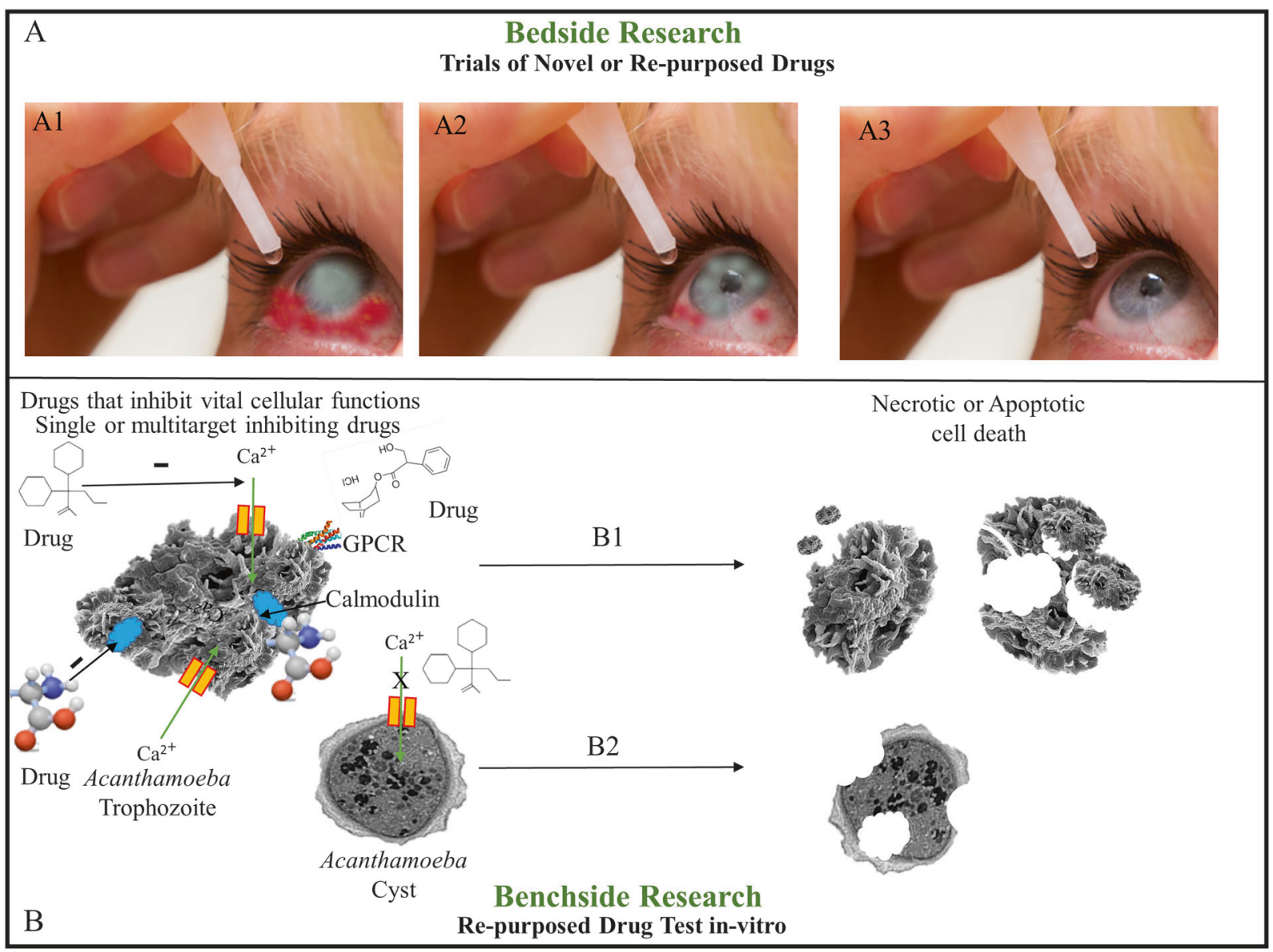

Fig. 2 Shows the schematic phases in testing novel or repurposed drugs for their effects in AK. A progressive improvement in AK (A2A3) without any adverse effects on the surrounding ocular tissue is an ideal output expected with the novel or repurposed drug. b Shows the recently reported receptors and proteins in Acanthamoeba (see text)

ophthalmologists can play toward research in AK is of crucial significance as the above-mentioned clinical efficacy of the drugs tested and their ocular safety issues can only be gauged by an experienced professional in the field. The efforts of the clinical professionals and ophthalmologists have recently enabled the testing of orphan chemical agents like polyhexamethylene biguanide (PHMB) in AK. Similar projects like the orphan disease Acanthamoeba keratitis (ODAK), which presented and published its finding in 2018 has shown the effects of offlabel drugs when tested on bedside [5].

Future endeavors to attempt trails (Fig. 2a) with agents already approved by drug-regulating authorities [3] that had repeatedly exhibited in vitro amoebicidal and cysticidal effects in Acanthamoeba (Fig. 2b) could make a successful short-term treatment of AK a real possibility. As grants and funded research remains the backbone of any research project, it is high time that pharmaceutical giants turn their attention towards the consideration of funding research in corneal diseases in general and $\mathrm{AK}$ in particular. Funding that have been targeted with the drugs suggested to be tested in $\mathrm{AK}^{3}$. The drugs already approved for their use in humans like calmodulin inhibitors, $\mathrm{Ca}^{2+}$ channel blocker, and anti-muscarinic agents have shown to be amoebicidal (B1) and cysticidal for Acanthamoeba in in vitro assays

the bench-to-bedside research projects [5] remains the lifeline for clinical research in searching a cure with a shortterm drug regimen in AK.

\section{Conclusions and future research}

In our understanding of the pathogenesis of $\mathrm{AK}$ and the search for a short-term curative regimen, there are many hurdles, but realistically, there is a chance of winning over this notorious and die-hard disease by bridging the benchside to bedside. Until a curative short-term treatment becomes a real possibility, at present, public and healthcare professionals have to put their best efforts to prevent AK by raising awareness regarding the modes of acquisition of an Acanthamoeba infection. Basic questions like, 'why a warmth and nutrition-loving parasite prefers to erode and invade an avascular cornea in preference over other more vascular and nutritive tissues in the surrounding? or "What cellular targets in Acanthamoeba play a kingpin role in the 
proliferation and survival of this protist pathogen? are few of the numerous queries that need to be investigated in order to select a targeted drug therapy of a shorter duration in AK.

Funding and Acknowledgment This project has been partly funded by Aga Khan University, Pakistan. Author would like to thank Miss Preet Katyara for critical review and helpful discussions.

\section{Compliance with ethical standards}

Conflict of interest The authors declare that they have no conflict of interest.

\section{References}

1. Carnt N PhD, Hoffman JJ MBBS, Verma S, Hau S, Radford CF $\mathrm{PhD}$, Minassian DC, et al. Acanthamoeba keratitis: confirmation of the UK outbreak and a prospective case-control study identifying contributing risk factors. Br J Ophthalmol. 2018;102:1621-1628. Epub 2018 Sep 19. PubMed PMID: 30232172. https://www.ncbi. nlm.nih.gov/pubmed/30232172.

2. Lalitha P, Lin CC, Srinivasan M, Mascarenhas J, Prajna NV, Keenan JD, et al. Acanthamoeba keratitis in South India: a longitudinal analysis of epidemics. Ophthalmic Epidemiol. 2012;19:111-5.

3. Baig AM, Waliani N, Karim S. Drug target discovery methods in targeting neurotropic parasitic amoebae. ACS Chem Neurosci. 2018;9:162-4.

4. Baig AM, Rana Z, Tariq S, Lalani S, Ahmad HR. Traced on the timeline: discovery of acetylcholine and the components of the human cholinergic system in a primitive unicellular eukaryote Acanthamoeba spp. ACS Chem Neurosci. 2018;;9:494-504. https://doi.org/10.1021/acschemneuro.7b00254.

5. J Gueudry, R Razakandrainibe, C Humbert, A Aifa, A Francois, LLe Goff, et al. Orphan drug for Acanthamoeba keratitis (ODAK) project: validation of an Acanthamoeba polyphaga keratitis model. Acta Ophthalmologica. 2014; 92: SN. 1755-375X; https://doi.org/ 10.1111/j.1755-3768.2014. 4421.x. 